Pacific Journal of Mathematics

GAME THEORETIC PROOF THAT CHEBYSHEV 


\section{GAME THEORETIC PROOF THAT CHEBYSHEV INEQUALITIES ARE SHARP}

\section{Albert W. Marshall aNd Ingram Olkin}

1. Summary. This paper is concerned with showing that Chebyshev inequalities obtained by the standard method are sharp. The proof is based on relating the bound to the solution of a game. An optimum strategy yields a portion of the extremal distribution, and the remainder is obtained as a solution of the relevant moment problem.

2. Introduction. Let $X$ be a random vector taking values in $\mathscr{X} \subset R^{k}$, and suppose that $E f(X) \equiv E\left(f_{1}(X), \cdots, f_{r}(X)\right)=\left(\varphi_{1}, \cdots, \varphi_{r}\right)$ $\equiv \phi$ is given, where $f_{j}$ is a real valued function on $\mathscr{Z}$. For convenience, we suppose $f_{1} \equiv 1$. An upper bound for $P\{X \in \mathscr{T}\}, \mathscr{T} \subset \mathscr{X}$, may be obtained as follows. If $a=\left(a_{1}, \cdots, a_{r}\right) \in R^{r}$ and $\chi_{\mathscr{F}}$ is the indicator of $\mathscr{T}$ then $a f^{\prime} \geqq \chi \mathscr{F}$ on $\mathscr{X}$ implies $P\{X \in \mathscr{T}\} \leqq a \mathscr{Q}^{\prime}$, and if $\mathscr{A}_{0}=\left\{a: a f^{\prime} \geqq\right.$ $\chi \mathscr{F}$ on $\mathscr{P}\}$, a "best" bound is given by

$$
P\{X \in \mathscr{T}\} \leqq \inf _{a \in \mathscr{A}_{0}} a \varphi^{\prime}
$$

In general, a bound is called sharp if it cannot be improved. For some cases, when $\mathscr{T}$ is assumed to be closed, the bound can actually be attained by a distribution satisfying the moment hypotheses.

The main result of this paper is

THEOREM 2.1. Inequality (2.1) is sharp in the following cases.

( I ) $X=\left(X_{1}, \cdots, X_{k}\right)$ with $E X_{i} X_{j}$ or $E X_{i}$ and $E X_{i} X_{j}$ given, $i, j=1, \cdots, k$.

(II) $X$ has range $(-\infty, \infty),[0, \infty)$, or $[0,1]$, and $E X^{j}$ is given, $j=1, \cdots, m$.

(III) $X$ is a random angle in $[0,2 \pi)$ and the trigonometric moments $E e^{i \alpha X}, \alpha= \pm 1, \cdots, \pm m$ are given.

Sharpness has been shown in (I) by Marshall and Olkin [6] when $\mathscr{T}$ is convex, and by Isii $[3,4]$ in the unbounded cases of (II). Sharpness has also been proved in a number of specialized situations.

In $\S 3$ the proof for (I) will be given in detail. The necessary alterations for each of the remaining cases will be given in $\S 4,5,6,7$. The solution of certain moment problems depend on conditions on Hankel matrices, i.e., matrices of the form $H=\left(h_{i+j}\right)$, and some results concerning these matrices are given in $\S 8$.

Received August 31, 1960. Research sponsored by the Office of Naval Research at Stanford University. 
The notation $A>0(\geqq 0)$ is used to mean that the matrix $A$ is symmetric and positive definite (p.s.d).

3. The multivariate case. The relation between inequality (2.1) and a game can be greatly simplified if we use matrix theoretic arguments. This is true in part because functions of the form $a f^{\prime}, a \in \mathscr{A}_{0}$, can be written very naturally as quadratic or bilinear forms.

Let $X=\left(X_{1}, \cdots, X_{k}\right)$ be a random vector on $R^{k}$ with $E X=\mu$ and moment matrix $E X^{\prime} X=\Sigma$. If $u \equiv u(x)=(1, x)$ for $x \in R^{k}$, then $E u^{\prime}(X)$ $u(X)=\left(\begin{array}{ll}1 & \mu \\ \mu^{\prime} & \Sigma\end{array}\right)=\Pi$. We assume $\Pi>0$, for otherwise the dimensionality of $X$ can be reduced.

Functions of the form $a f^{\prime}, a \in \mathscr{A}_{0}$ can be written as $u A u^{\prime}, A$ : $k+1 \times k+1, A \in \mathscr{A}=\left\{A ; A \geqq 0, u A u^{\prime} \geqq 1\right.$ for $\left.x \in \mathscr{T}\right\}$. Hence

$$
P\{X \in \mathscr{T}\} \leqq \inf _{a \in \mathscr{A}_{0}} a \mathscr{Q}^{\prime}=\inf _{A \in \mathscr{A}} \operatorname{tr} A \Pi .
$$

Let $x_{1}, \cdots, x_{m}$ be points (row vectors) in $R^{k}, u_{i}=u\left(x_{i}\right), p_{1}, \cdots, p_{m}$, $\Sigma p_{i}=1$ be probabilities, $T=\left(u_{1}^{\prime}, \cdots, u_{m}^{\prime}\right), D_{p}=\operatorname{diag}\left(p_{1}, \cdots, p_{m}\right)$, and $H=T D_{p} T^{\prime}$. By $H \sim \mathscr{T}$ we mean that all $x_{i} \in \mathscr{T}$. The condition $u A u^{\prime} \geqq 1$ for $x \in \mathscr{T}$ can then be written as $\operatorname{tr} A H \geqq 1$ for $H \sim \mathscr{T}$, so that $\mathscr{A}=\{A: A \geqq 0, \operatorname{tr} A H \geqq 1$ for $H \sim \mathscr{T}\}$.

With this notation, we can rewrite the bound (3.1) in a form which is suggestive of a game.

$$
\begin{aligned}
& \inf _{A \in \mathscr{A}} \operatorname{tr} A \Pi=\inf _{\substack{\{A: \inf \operatorname{tr} A H \geqq 1, A \geqq 0\} \\
H \sim \mathscr{T}}} \operatorname{tr} A \Pi \\
& =\inf _{S \geqq 0}\left(\frac{\operatorname{tr} S \Pi}{\inf _{H \sim \mathscr{T}} \operatorname{tr} S H}\right)=\left(\sup _{S \geqq 0} \inf _{H \sim \mathscr{T}} \frac{\operatorname{tr} S H}{\operatorname{tr} S I I}\right)^{-1} \\
& =\left(\sup _{\{S: S \geqq 0, \operatorname{tr} S H \leqq 1\}} \inf _{I I \sim \mathscr{T}} \operatorname{tr} S H\right)^{-1} \equiv \nu^{-1} .
\end{aligned}
$$

In view of (3.2) it is natural to consider the game $G=(\mathscr{S}, \mathscr{H}, g)$, where $\mathscr{S}=\{S: S \geqq 0, \operatorname{tr} S \Pi \leqq 1\}$ and $\mathscr{H}=\{H: H \sim \mathscr{T}\}$ are the strategy spaces for players I and II, respectively, and $g(S, H)=\operatorname{tr} S H$ is the payoff to player I.

Clearly $\mathscr{S}$ and $\mathscr{H}$ are closed and convex. Further, $\mathscr{S}$ is bounded since

$$
\|S\|^{2} \equiv\left(\operatorname{tr} S S^{\prime}\right) \leqq(\operatorname{tr} S) c_{M}(S) \leqq(\operatorname{tr} S)^{2} \leqq(\operatorname{tr} S \Pi)^{2} / c_{m}^{2}(\Pi) \leqq 1 / c_{m}^{2}(\Pi),
$$

where $c_{m}(A), c_{m}(A)$ are the minimum and maximum characteristic roots of $A$. For the present we assume that $\mathscr{P}$ is bounded, then by [2, Section 2.5], $G$ has a value and there exist optimal strategies $S_{0} \in \mathscr{S}, H_{0} \in \mathscr{H}$, such that 


$$
\operatorname{tr} S H_{0} \leqq \operatorname{tr} S_{0} H_{0}=\nu \leqq \operatorname{tr} S_{0} H, \quad \text { for all } S \in \mathscr{S}, H \in \mathscr{H} .
$$

The optimal strategy $S_{0}$ has the property that $\inf A \in \mathscr{A} \operatorname{tr} A \Pi=\operatorname{tr} A_{0} \Pi$, where $A_{0}=S_{0} / \nu$.

To prove sharpness of (3.1), we must show that there exists a distribution for $X$ such that $P\{X \in \mathscr{T}\}=1 / \nu$, and $E u^{\prime} u=\Pi . H_{0}$ is the moment matrix of a distribution $F_{1}$ on points in $\mathscr{T}$. If we can prove the existence of a probability distribution $F$ for $X$ of the form $F=$ $F_{1} / \nu+F_{2}$, and with moment matrix $\Pi$, then this distribution attains equality in (3.1). To see this, note that $F$ assigns at least probability $\nu$ to $\mathscr{T}$, and by (3.1) it can assign at most probability $\nu$ to $\mathscr{T}$.

To show the above, we need only show that a distribution $F_{2}$ exists with total variation $1-1 / \nu$ and moment matrix $\Psi=\Pi-H_{0} / \nu$. The following Lemma yields this result.

LemMA 3.1. Let $\Pi>0, \mathscr{S}=\{S: S \geqq 0, \operatorname{tr} S \Pi \leqq 1\}$.

(i) If $\operatorname{tr} S H \leqq \nu$ for all $S \in \mathscr{S}$, then $\Psi=\Pi-H / \nu \geqq 0$.

(ii) If $\operatorname{tr} S H=\nu$ for some $S_{0} \in \mathscr{S}$, then $\Psi$ is not strictly $>0$.

(iii) If $\operatorname{tr} S H<\nu$ for all $S \in \mathscr{S}$, then $\Psi>0$.

Proof. There exists a representation $I=W W^{\prime}, H=W D_{\theta} W^{\prime}$, $|W| \neq 0, D_{\theta}=\operatorname{diag}\left(\theta_{0}, \cdots, \theta_{k}\right)$, and hence $\Psi \geqq 0$ if and only if $\theta_{i} \leqq \nu$, $i=0, \cdots, k$. If $W^{\prime} S W=\left(\begin{array}{ll}1 & 0 \\ 0 & 0\end{array}\right)$, then $S \in \mathscr{S}$, and from $\operatorname{tr} S H=$ $\operatorname{tr} W^{\prime} S W D_{\theta} \leqq \nu$, we obtain $\theta_{0} \leqq \nu$. Part (i) follows using permutations. If $\operatorname{tr} S H<\nu$, then in the above argument, each $\theta_{i}<\nu$. If $\operatorname{tr} S_{0} H=$ $\operatorname{tr}\left(W^{\prime} S_{0} W\right) D_{\theta}=\nu$ and $\operatorname{tr} W^{\prime} S_{0} W \leqq 1$, then at least one of the $\theta_{i}$ is equa to $\nu$.

The condition that $\mathscr{H}$ be bounded now can be removed, since $\left\|H_{0}\right\|^{2} \leqq\left(\operatorname{tr} H_{0}\right)^{2} \leqq[\nu \operatorname{tr} I I]^{2}$, by Lemma 3.1 .

REMARK 3.1. We note that $\operatorname{tr} S_{0} I=1$, for if not, $\alpha S_{0}$ for $\alpha>1$ would violate (3.3).

$S_{0}$ and $H_{0}$ are related by $\nu S_{0} I=S_{0} H_{0}$. This follows from the fact that $\operatorname{tr} S_{0} \Psi=\operatorname{tr} S_{0}\left(\Pi-H_{0} / \nu\right)=0$ and $\Psi \geqq 0$ implies that $S_{0}^{1 / 2} \Psi S_{0}^{1 / 2}=0$, or equivalently that $S_{0}^{1 / 2} \Psi^{1 / 2}=0$, which yields the result.

REMARK 3.2. In the above development we assumed that $E X=\mu$ was given. If this is not the case, then choose $\mathscr{S}=\left\{S=\left(\begin{array}{ll}\alpha & 0 \\ 0 & S_{1}\end{array}\right): S>0\right.$, $\operatorname{tr} S I I 1\}, S_{1}: k \times k$, and the entire development remains unchanged with $S_{1}$ replacing $S$, since $S \geqq 0$ if and only if $\alpha>0, S_{1} \geqq 0$ and $\operatorname{tr} S \Pi=$ $\alpha+\operatorname{tr} S_{1} \Sigma$.

We now summarize the essential points of the proof which are appropriately modified in each of the remaining cases. 
(1) Introduce vectors $u(x)$ and $v(x)(u=v$ in the above) such that (i) $E v^{\prime}(X) u(X)=I I$ is a matrix of given moments, (ii) $a f^{\prime}, a \in \mathscr{A}_{0}$ can be written as $u A v^{\prime}$ with $A \in \mathscr{A}$. To define $\mathscr{A}$ we first must characterize $\mathscr{A}_{0}$.

(2) Define $\mathscr{H}$, a set of moment matrices of the same kind as $I$, but corresponding to distributions on $\mathscr{T}$.

(3) Define $\mathscr{S}$ and show that $\mathscr{S}$ is bounded.

(4) Use the game to assert that $H_{0}$ exists, and show that the moment problem with moments defined by $\Psi=\Pi-H_{0} / \nu$ has a solution with $\psi_{11}=1-1 / \nu$.

4. Univariate distributions on $(-\infty, \infty)$. Let $u(x)=v(x)=(1, x$, $\left.\cdots, x^{n}\right)$. Then polynomials $a f^{\prime}(x)$ of degree $\leqq 2 n$ which are nonnegatve in $(-\infty, \infty)$ can be expressed as $u A u^{\prime}, A \geqq 0,[7$, p. 82]. Hence $\mathscr{A}=$ $\left\{A: A \geqq 0, u A u^{\prime} \geqq 1\right.$ for $\left.x \in \mathscr{T}\right\}$, and (3.1) holds. Note that $\Pi=\left(\pi_{i+j-2}\right)=$ $\left(E X^{i+j-2}\right), i, j=1, \cdots, n+1$. Let $-\infty<t_{i}<\infty, u_{i}=u\left(t_{i}\right), i=1, \cdots, m$, $T=\left(u_{1}^{\prime}, \cdots, u_{m}^{\prime}\right), \quad D_{p}=\operatorname{diag}\left(p_{1}, \cdots, p_{m}\right) \geqq 0, \quad \operatorname{tr} D_{p}=1, \quad H=T D_{p} T^{\prime}=$ $\left(h_{i+j-2}\right), i, j=1, \cdots, n+1$. Define $\mathscr{H}=\left\{H: t_{i} \in \mathscr{T}, i=1, \cdots, m\right\}, \mathscr{P}=$ $\{S: S \geqq 0, \operatorname{tr} S \Pi \leqq 1\}$. We assume that the moment problem corresponding to the given moments $\left\{\pi_{0}, \cdots, \pi_{2 n}\right\}$ is not determined so that $I I>0$, [8, Th. 3.3], and the previous argument that $\mathscr{P}$ is bounded holds. Assuming that $\mathscr{S} C$ is bounded, there exists an $S_{0}$ and $H_{0}=\left(h_{i+j-2}^{\circ}\right)$ satisfying (3.3), and with Lemma 3.1 we conclude as before that the boundedness condition on $\mathscr{Y}$ can be removed.

Since $\pi_{0}=h_{0}^{0}=1, \psi_{0}=1-1 / \nu$. Define $\Delta_{r}=\left|\psi_{i+j-2}\right|_{i, j=1}^{r+1}$; then since $\Psi \geqq 0$, by Theorem 8.1 it follows that $\Delta_{1}>0, \cdots, \Delta_{r-1}>0, \Delta_{r}=0, \cdots$, $\Delta_{n}=0$, for some $r$. The reduced (Hamburger) moment problem has a solution if and only if $\Psi \geqq 0$, in which case there exists a (unique) representation $\psi_{j}=\sum_{i=1}^{r} p_{\imath} \xi_{\imath}^{j}, j=0,1, \cdots, 2 n-1$, and $\psi_{2 n}=\sum_{r=1}^{r} p_{i} \xi_{i}^{2 n}+$ $c, c \geqq 0$, and $c=0$ if $r=n,[8$, p. 85].

In the event $c>0$, by using an $\varepsilon$-good strategy for player II to guarantee $\Psi$ strictly $>0$, we obtain a distribution with moments $\left\{\pi_{0}, \cdots\right.$, $\left.\pi_{2 n}\right\}$, which assigns probability $1 /(\nu+\varepsilon)$ to $\mathscr{T}$.

REMARK 4.1. The representation obtained from [7, p. 82] is of the form $\left(\Sigma u_{i} c_{i}\right)^{2}+\left(\Sigma u_{2} d_{i}\right)^{2}$, which is expressible as $u A u^{\prime}$, where $A=c^{\prime} c+d^{\prime} d$. However, the same class of polynomials is obtained if we include all $A \geqq 0$.

REMARK 4.2. If $\mathscr{S}$ is bounded, there exists an extremal distribution with a spectrum consisting of at most $2(n+1)$ points. This follows from the fact that the least number of points contributing to $H_{0}$ is at most $(n+1),[2, \S 2.5]$, and to $\Psi$ is at most $(n+1)$ points by the previous argument. 
5. Univariate Case on $[0, \infty)$. Consider first the case $m=2 n-1$, and let $u(x)=\left(1, x, \cdots, x^{n-1}\right), v(x)=\left(1, x, \cdots, x^{n}\right)$. Then polynomials $a f^{\prime}(x)$ of degree $\leqq 2 n-1$ can be expressed as $u[(B, 0)+(0, C)] v^{\prime} \equiv u A v^{\prime}$, where $B \geqq 0, C \geqq 0$ are $n \times n$ matrices (See [7, p. 82] and Remark 4.1). Hence $\mathscr{A}=\left\{A: B \geqq 0, C \geqq 0, u A v^{\prime} \geqq 1\right.$ for $\left.x \in \mathscr{T}\right\}$, and (3.1) holds. Now $\Pi=\left(\pi_{i+j-2}\right)=\left(E X^{i+j-2}\right), i=1, \cdots, n+1 ; j=1, \cdots, n$. Let $0<t_{i}<\infty$, $u_{i}=u\left(t_{i}\right), v_{i}=v\left(t_{i}\right), i=1, \cdots, m, \quad T_{1}=\left(u_{1}^{\prime}, \cdots, u_{m}^{\prime}\right), \quad T_{2}=\left(v_{1}^{\prime}, \cdots, v_{m}^{\prime}\right)$, $D_{p}=\operatorname{diag}\left(p_{1}, \cdots, p_{m}\right) \geqq 0, \operatorname{tr} D_{p}=1, H=T_{1} D_{p} T_{2}^{\prime}=\left(h_{i+j-2}\right), i=1, \cdots, n+$ $1 ; j=1, \cdots, n$. Define $\mathscr{H}=\left\{H: t_{i} \in \mathscr{T}, i=1, \cdots, m\right\}, \mathscr{S}=\{S=$ $\left.\left(S_{1}, S_{2}\right): S_{1} \geqq 0, S_{2} \geqq 0, \operatorname{tr}\left[\left(S_{1}, 0\right)+\left(0, S_{2}\right)\right] \Pi \leqq 1\right\}, S_{1}, S_{2}: n \times n, 0: n \times 1$. Assuming that the moment problem corresponding to $\Pi$ is not determined, i.e., $\Pi_{(1)}=\left(\pi_{i+j-2}\right), i, j=1, \cdots, n, \Pi^{(1)}=\left(\pi_{i+j-1}\right), i, j=1, \cdots, n$, are positive definite, $[8$, p. 6$]$, the argument of $\S 3$ that $\mathscr{S}$ is bounded holds, with $\|S\| \equiv\left\|\left(S_{1}, 0\right)+\left(0, S_{2}\right)\right\|$.

Assuming that $\mathscr{C}$ is bounded, there exists an $S_{0}=\left(S_{10}, 0\right)+\left(0, S_{20}\right)$ and $H_{0}=\left(h_{i+j-2}^{0}\right), i=1, \cdots, n+1 ; j=1, \cdots, n$, satisfying (3.3). Define $H_{0(1)}$ and $H_{0}^{(1)}$ in the some manner as $\Pi_{(1)}$ and $\Pi^{(1)}$. An application of Lemma 3.1 yields $\Psi_{(1)}=\Pi_{(1)}-H_{0(1)} / \nu \geqq 0$ and $\Psi^{(1)}=\Pi^{(1)}-H_{0}^{(1)} / \nu \geqq 0$. The boundedness condition of $\mathscr{K}$ can now be removed since $\left\|H_{0}\right\|^{2} \leqq$ $\left\|H_{01}\right\|^{2}+\left\|H_{0}^{(1)}\right\|^{2} \leqq \nu \operatorname{tr}\left(\Pi_{(1)}+\Pi^{(1)}\right)$. Also $\psi_{0}=\pi_{0}-h_{0} / \nu=1-1 / \nu$.

In order for the reduced (Stieltjes) moment problem to have a solution, it is necessary that both $\Psi_{(1)}$ and $\Psi^{(1)}$ be $\geqq 0 .^{1}$

Recall from $\S 4$ that $\Delta_{r}=\left|\psi_{i+j-2}\right|, i, j=1, \cdots, r+1$. Now define $\Delta_{r}^{(1)}=\left|\psi_{i+j-1}\right|, i, j=1, \cdots, r+1$. From Theorem 8.1 it follows that either

(i ) $\Delta_{0}>0, \cdots, \Delta_{r}>0, \Delta_{r+1}=\cdots=\Delta_{n}=0$ and $\Delta_{0}^{(1)}>0, \cdots, \Delta_{r}^{(1)}>$ $0, \Delta_{r+1}^{(1)}=\cdots=\Delta_{n}^{(1)}=0$, or

(ii) $\Delta_{0}>0, \cdots, \Delta_{r}>0, \quad \Delta_{r+1}=\cdots=\Delta_{n}=0$ and $\quad \Delta_{0}^{(1)}>0, \cdots$, $\Delta_{r-1}^{(1)}>0, \Delta_{r}^{(1)}=\cdots=\Delta_{n}^{(1)}=0$, for some $r$. But these are the conditions that there exist a distribution whose spectrum consists of $r+1$ points distinct from 0 in case (i) and including 0 in (ii).

If $m=2 n$, let $u(x)=v(x)=\left(1, x, \cdots, x^{n}\right)$. Then polynomials $a f^{\prime}(x)$ of degree $\leqq 2 n$ can be expressed as $v\left[B+\left(\begin{array}{ll}0 & C \\ 0 & 0\end{array}\right)\right] v^{\prime}$, where $B: n+1 \times$ $n+1, C: n \times n, B \geqq 0, C \geqq 0,[7$, p. 82]. The remainder of the proof is essentially the same as for the case $m=2 n-1$ above.

6. Univariate distribution on $[0,1]$. We first deal with the case when an odd number of moments is given. Let $u(x)=\left(1, x, \cdots, x^{n-1}\right)$, $v(x)=\left(1, x, \cdots, x^{n}\right) . \quad$ Now $\quad I=\left(\pi_{i+j-2}\right)=\left(E X^{i+j-2}\right), i=1, \cdots, n+1$; $j=1, \cdots, n$. Then polynomials $a f^{\prime}(x)$ of degree $\leqq 2 n-1$ which are

1 This result was communicated to the authors by S. Karlin. The proof is similar to that for the reduced Hausdorff moment problem given in [5]. 
nonnegative in $[0,1]$ can be expressed as $u[(B, 0)+(0, C-B)] v^{\prime} \equiv u A v^{\prime}$, where $B$ and $C$ are $n \times n$ matrices, $B \geqq 0, C \geqq 0$, (See [7, p. 82] and Remark 4.1). Hence $\mathscr{A}=\left\{A: B \geqq 0, C \geqq 0, u A v^{\prime} \geqq 1\right.$ for $\left.x \in \mathscr{T}\right\}$, and (3.1) holds. We assume that the moment problem corresponding to the given moments $\left\{\pi_{0}, \cdots, \pi_{2 n-1}\right\}$ is not determined. This means that $\Pi^{(1)}=$ $\left(\pi_{i+j-1}\right), i, j=1, \cdots, n$, and $\Pi_{(2)}=\left(\pi_{i+j-2}-\pi_{i+j-1}\right), i, j=1, \cdots, n$, are both positive definite, [5, p. 55] or [8, p.77]. (In the latter reference the conditions are presented for the interval $[-1,1]$.)

Let $0 \leqq t_{i} \leqq 1, u_{i}=u\left(t_{i}\right), v_{i}=v\left(t_{i}\right), i=1, \cdots, m, T_{1}=\left(u_{1}^{\prime}, \cdots, u_{m}^{\prime}\right)$, $T_{2}=\left(v_{1}^{\prime}, \cdots, v_{m}^{\prime}\right), \quad D_{p}=\operatorname{diag}\left(p_{1}, \cdots, p_{m}\right) \geqq 0, \quad \operatorname{tr} D_{p}=1, \quad H=T_{1} D_{p} T_{2}^{\prime}=$ $\left(h_{i+j-2}\right), i=1, \cdots, n+1 ; j=1, \cdots, n$. Define $\mathscr{\mathscr { C }}=\left\{H: t_{i} \in \mathscr{T}, i=1\right.$, $\cdots, m\}, \mathscr{S}=\left\{\left(S_{1}, S_{2}\right): S_{1} \geqq 0, S_{2} \geqq 0, \operatorname{tr}\left[\left(S_{1} 0\right)+\left(0, S_{2}-S_{1}\right)\right] \Pi \leqq 1\right\}$. We first show that $\mathscr{S}$ is bounded:

$$
\|S\|^{2} \equiv\left\|\left(S_{1}, 0\right)+\left(0, S_{2}-S_{1}\right)\right\| \leqq 2 \operatorname{tr} S_{1}^{2}+\operatorname{tr} S_{2}^{2} \leqq 2\left(\operatorname{tr} S_{1}\right)^{2}+\left(\operatorname{tr} S_{2}\right)^{2} .
$$

But $\operatorname{tr} S I I=\operatorname{tr} S_{1} \Pi_{(2)}+\operatorname{tr} S_{2} \Pi^{(1)} \leqq 1$, and $\Pi_{(2)}>0, \quad \Pi^{(1)}>0$, so that $\operatorname{tr} S_{1} \leqq 1 / c_{m}\left(\Pi_{(2)}\right), \operatorname{tr} S_{2} \leqq 1 / c_{m}\left(\Pi^{(1)}\right)$, and $\mathscr{S}$ is bounded.

Assuming that $\mathscr{\mathcal { C }}$ is bounded, there exists an $S_{0}=\left(S_{10}, 0\right)+\left(0, S_{20}-S_{10}\right)$ and $H_{0}=\left(h_{i+j-2}^{0}\right), i=1, \cdots, n+1 ; j=1, \cdots, n$, satisfying (3.3). Define $H_{0(2)}$ and $H_{0}^{(1)}$ as for $\Pi_{(2)}$ and $\Pi^{(1)}$; then an application of Lemma 3.1 yields

$$
\Psi_{(2)}=\Pi_{(2)}-H_{0(2)} / \nu \geqq 0, \Psi^{(1)}=\Pi^{(1)}-H_{0}^{(1)} / \nu \geqq 0 .
$$

The boundedness condition on $\mathscr{H}$ can now be removed since $\left\|H_{0}\right\|^{2} \leqq$ $2\left\|H_{0(2)}\right\|^{2}+2\left\|H_{0}^{(1)}\right\|^{2} \leqq \nu \operatorname{tr}\left(\Pi_{(2)}+\Pi^{(1)}\right)$. Also $\psi_{0}=\pi_{0}-h_{0} / \nu=1-1 / \nu$.

In order for the reduced (Hausdorff) moment problem to have a solution, it is necessary that both $\Psi_{(2)}$ and $\Psi^{(1)}$ be $\geqq 0$, [5, p. 55].

If an even number of moments is given, we let $u(x)=v(x)=(1, x$, $\left.\cdots, x^{n}\right)$. Now $I=\left(\pi_{i+j-2}\right), i, j=1, \cdots, n+1$. Polynomials $a f^{\prime}(x)$ of degree $\leqq 2 n$ which are nonnegative in $[0,1]$ can be expressed as $u\left[\left(\begin{array}{ll}B & 0 \\ 0 & 0\end{array}\right)+\left(\begin{array}{ll}0 & C \\ 0 & 0\end{array}\right)+\left(\begin{array}{rr}0 & 0 \\ 0 & -C\end{array}\right)\right] u^{\prime} \equiv u A u^{\prime}$, where $B$ and $C$ are $n \times n$ matrices, $B \geqq 0, C \geqq 0$, (See [7, p. 82] and Remark 4.1). Hence $\mathscr{A}=$ $\left\{A: B \geqq 0, C \geqq 0, u A u^{\prime} \geqq 1\right.$ for $\left.x \in \mathscr{T}\right\}$, and (3.1) holds. We assume that the moment problem corresponding to the given moments $\left\{\pi_{0}, \cdots\right.$, $\left.\pi_{2 n}\right\}$ is not determined. This means that $I$ and $\Pi_{(3)}=\left(\pi_{i+j-1}-\pi_{i+j}\right)$, $i, j=1, \cdots, n$, are positive definite, [5, p. 55] or [8, p. 77].

The remainder of the argument is analogous to the odd moment case.

REMARK 6.1. As in Remark 4.1, if $\mathscr{T}$ is bounded, there exists an extremal distribution with a spectrum consisting of at most $2(n+1)$ points. This follows from $[2, \S 2.5]$ and $[5, \S 17]$. 
REMARK 6.2. A condition for the solution of the Hausdorff moment problem with an infinite number of moments is the condition that

$$
\Delta^{k} \mu_{j}=\mu_{j}-\left(\begin{array}{l}
k \\
1
\end{array}\right) \mu_{j+1}+\left(\begin{array}{l}
k \\
2
\end{array}\right) \mu_{j+2}+\cdots+(-1)^{k} \mu_{j+k} \geqq 0, \quad k, j=0,1, \cdots
$$

However, this condition with $k, j=0,1, \cdots, n$ is not sufficient for a solution of the reduced moment problem. It is interesting to note that this condition enters naturally using an alternative formulation. Polynomials $a f^{\prime}(x)$ which are nonnegative in $[0,1]$ may be represented as $\Sigma a_{i j}(1-x)^{i} x^{j}$, where $a_{i j} \geqq 0$. If we let $u(x)=\left(1,(1-x), \cdots,(1-x)^{n}\right)$, $v(x)=\left(1, x, \cdots, x^{n}\right)$, then the representation is $u A v^{\prime}, a_{i j} \geqq 0$. Now $\Pi=\left(E(1-X)^{i-1} X^{j-1}\right)=\left(\Delta^{i-1} \mu_{j-1}\right), i, j=1, \cdots, n+1$. Using a similar development as before, $\mathscr{S}=\left\{S: s_{i j} \geqq 0, \operatorname{tr} S \Pi \leqq 1\right\}$, and from Lemma $3.1, \Psi=\Pi-H_{0} / \nu=\left(\Delta^{i-1} \mu_{j-1}\right)-\left(\Delta^{i-1} \mu_{j-1} / \nu\right) \geqq 0$. Let $\psi_{j}=\mu_{j}-h_{j} / \nu$, $\Psi=\left(\Delta^{i-1} \psi_{j-1}\right)$; we wish to show that $\Delta^{i-1} \psi_{j-1} \geqq 0$. By choosing $S$ to have all zeros except $s_{i j}=1 / \Delta^{i-1} \pi_{j-1}, \operatorname{tr} S \Pi=1$. The result follows after using (3.3).

7. Random angle in $[0,2 \pi)$. If $u(x)=v(x)=\left(1, e^{\text {inx }}, \cdots, e^{\text {inx }}\right)$, then polynomials $a f^{\prime}(x)$ which are nonnegative in $[0,2 \pi)$ can be expressed as $u A u^{\prime}, A \geqq 0$, (See [7, p. 82] and Remark 4.1). Hence $\mathscr{A}=\{A: A \geqq 0$, $u A u^{\prime} \geqq 1$ for $\left.x \in \mathscr{T}\right\}$, and (3.1) holds. Now $\Pi=\left(\pi_{j-k}\right)=\left(E e^{i(j-k) X}\right)$, $j, k=1, \cdots, n+1$.

The proof is virtually that of $\S 4$, noting only that the reduced trigonometric (Herglotz) moment problem has a solution if the Toeplitz matrix $\Pi>0$. (See footnote, $\S 5$.)

7.1. An example. The authors are unaware of any Chebyshev inequalities when trigonometric moments are available, and we present a simple illustration.

Theorem 7.1. If $X$ is a random angle in $[0,2 \pi)$ and $E \sin X=\alpha$, $E \cos X=\beta$, then

$$
\begin{aligned}
& P\{2 \theta<X<2 \varphi\} \geqq 1-\frac{1-\alpha \sin (\theta+\varphi)-\beta \cos (\theta+\varphi)}{1-\cos (\phi-\theta)}, \\
& P\{2 \theta \leqq X \leqq 2 \varphi\} \leqq \frac{1+\alpha \sin (\theta+\varphi)+\beta \cos (\theta+\varphi)}{1+\cos (\varphi-\theta)}, \\
& \quad 0 \leqq \theta \leqq \varphi \leqq \pi
\end{aligned}
$$

Proof. Choose $f(x)=c_{1}+c_{2} \sin x+c_{3} \cos x$. The conditions $f(\theta+\phi)=0, \quad f(2 \theta)=f(2 \varphi)=1$ lead to (7.1), and the conditions $f(\theta+\phi+\pi)=0, f(2 \theta)=f(2 \varphi)=1$ lead to $(7.2)$. 
8. Properties of Hankel matrices. In this section we obtain several properties of Hankel matrices which were required in $\S \S 4$ and 5. These properties are known as a consequence of the solution of moment problems, but it may be of interest to present matrix theoretic proofs. We need the following preliminaries.

A matrix $U=\left(u_{i+j-2}\right), i, j=1, \cdots, n$ is called a Hankel matrix. By the $r$ th compound, $A^{(r)}$, of a matrix $A: n \times n$ we mean the matrix whose elements are the $r$ th order minors of $A$ arranged in lexicographic order; thus $A^{(r)}:\left(\begin{array}{l}n \\ r\end{array}\right) \times\left(\begin{array}{l}n \\ r\end{array}\right)$. The following properties of compound matrices are well-known, e.g., [1].

(8.1) Let $A$ be symmetric. The characteristic roots of $A^{(r)}$ are the $\left(\begin{array}{l}n \\ r\end{array}\right)$ products of $r$ characteristic roots of $A$. Thus, $A^{(r)} \geqq 0$ if and only if $A \geqq 0$.

$$
\left|A^{(r)}\right|=|A|^{\left(\begin{array}{c}
n-1 \\
r-1
\end{array}\right)} .
$$

THeORem 8.1. If the Hankel matrix $U=\left(u_{i+j-2}\right), i, j=1, \cdots$, $r+1$, is $\geqq 0$, and if $\Delta_{r}=\left|u_{i+j-2}\right|_{i, j=1}^{r}=0$, then $\Delta_{r+1}=0$.

Proof. Suppose $u_{0}=0$, then by nonnegativity of each $2 \times 2$ principal minor, it follows that $u_{0}=u_{1}=\cdots=u_{2 n-1}=0, u_{2 n} \geqq 0$. But $U^{(r)} \geqq 0$ has first element 0 , and hence its first row is 0 , so that $\Delta_{r}=0$.

TheOREM 8.2. Let $U=\left(u_{i+j-2}\right), \quad i, j=1, \cdots, r+1, \quad V=\left(u_{i+j-1}\right)$, $i, j=1, \cdots, r+1, \quad U \geqq 0, V \geqq 0$. Then $\Delta_{r}=0 \Rightarrow \Delta_{r}^{(1)}=0 \Rightarrow \Delta_{r+1}=0$, where $\Delta_{m}=\left|u_{i+j-2}\right|, i, j=1, \cdots, m ; \Delta_{m}^{(1)}=\left|u_{i+j-1}\right|, i, j=1, \cdots, m$.

Proof. In the $r$ th compound $U^{(r)}, \Delta_{r}=u_{11}^{(r)}=0$ implies that $u_{12}^{(r)}=$ $\Delta_{r}^{(1)}=0$. In the $r$ th compound $V^{(r)}, \Delta_{r}^{(1)}=v_{11}^{(r)}=0$, and hence all $v_{i j}^{(r)}=0$, except possibly the last diagonal element, which is a function of $u_{2 r+1}$. In $U^{(r+1)}$, the last column does not depend on $u_{2 r+1}$, and its elements are the $v_{i j}^{(r)}$ which are zero. Hence $\left|U^{(r+1)}\right|=0$, so that $\Delta_{r+1}=0$.

9. Acknowledgment. We are grateful to Herman Rubin for some valuable discussions. He also pointed out that sharpness of Chebyshev inequalities can be proved quite generally without knowledge of moment problem solutions by an application of the Hahn-Banach extension theorem. However, the present proof provides considerable information concerning extremal distributions.

\section{REFERENCES}

1. A. C. Aitken, Determinants and matrices, 9th edition, Edinburgh: Oliver and Boyd, 1956. 
2. David Blackwell and M. A. Girshick, Theory of Games and Statistical Decisions, John Wiley and Sons, New York, 1954.

3. Keiiti Isii, On a method for generalizations of Tchebycheff's inequality, Ann. Inst. Stat. Math., 10 (1959), 65-88.

4. - Bounds on probability for non-negative random variables, Ann. Inst. Stat. Math., 11 (1959), 89-99.

5. S. Karlin and L. S. Shapley, Geometry of Moment Spaces, Memoirs Amer. Math. Soc., No. 12, 1953.

6. Albert W. Marshall and Ingram Olkin, Multivariate Chebyshev inequalities, Ann. Math. Stat., Vol. 31 (1960), 1001-1014.

7. G. Pólya and G. Szegö, Aufgaben und Lehrsätze aus der Analysis, Vol. II, $2^{\text {nd }}$ edition, Springer, Berlin, 1954.

8. J. A. Shohat and J. D. Tamarkin, The problem of moments, Mathematical Surveys Number 1, American Mathematical Society, 1943.

STANFORD UNIVERSITY AND BOEING SCIENTIFIC LABORATORIES;

UNIVERSITY OF MINNESOTA AND STANFORD UNIVESITY 



\section{PACIFIC JOURNAL OF MATHEMATICS}

\section{EDITORS}

\author{
RaLPh S. Phillips \\ Stanford University \\ Stanford, California \\ F. H. BRowNELL \\ University of Washington \\ Seattle 5 , Washington
}

A. L. Whiteman

University of Southern California

Los Angeles 7, California

L. J. Paige

University of California

Los Angeles 24, California

\author{
E. F. BECKENBACH \\ T. M. CHERRY
}

\author{
ASSOCIATE EDITORS

$\begin{array}{lll}\text { D. DERRY } & \text { H. L. ROYDEN } & \text { E. G. STRAUS } \\ \text { M. OHTSUKA } & \text { E. SPANIER } & \text { F. WOLF }\end{array}$

\section{SUPPORTING INSTITUTIONS}

\author{
UNIVERSITY OF BRITISH COLUMBIA \\ CALIFORNIA INSTITUTE OF TECHNOLOGY \\ UNIVERSITY OF CALIFORNIA \\ MONTANA STATE UNIVERSITY \\ UNIVERSITY OF NEVADA \\ NEW MEXICO STATE UNIVERSITY \\ OREGON STATE COLLEGE \\ UNIVERSITY OF OREGON \\ OSAKA UNIVERSITY \\ UNIVERSITY OF SOUTHERN CALIFORNIA
}

\author{
STANFORD UNIVERSITY \\ UNIVERSITY OF TOKYO \\ UNIVERSITY OF UTAH \\ WASHINGTON STATE COLLEGE \\ UNIVERSITY OF WASHINGTON \\ AMERICAN MATHEMATICAL SOCIETY \\ CALIFORNIA RESEARCH CORPORATION \\ HUGHES AIRCRAFT COMPANY \\ SPACE TECHNOLOGY LABORATORIES \\ NAVAL ORDNANCE TEST STATION
}

Mathematical papers intended for publication in the Pacific Journal of Mathematics should be typewritten (double spaced), and the author should keep a complete copy. Manuscripts may be sent to any one of the four editors. All other communications to the editors should be addressed to the managing editor, L. J. Paige at the University of California, Los Angeles 24, California.

50 reprints per author of each article are furnished free of charge; additional copies may be obtained at cost in multiples of 50 .

The Pacific Journal of Mathematics is published quarterly, in March, June, September, and December. The price per volume (4 numbers) is $\$ 12.00$; single issues, $\$ 3.50$. Back numbers are available. Special price to individual faculty members of supporting institutions and to individual members of the American Mathematical Society: $\$ 4.00$ per volume; single issues, $\$ 1.25$.

Subscriptions, orders for back numbers, and changes of address should be sent to Pacific Journal of Mathematics, 103 Highland Boulevard, Berkeley 8, California.

Printed at Kokusai Bunken Insatsusha (International Academic Printing Co., Ltd.), No. 6, 2-chome, Fujimi-cho, Chiyoda-ku, Tokyo, Japan.

\section{PUBLISHED BY PACIFIC JOURNAL OF MATHEMATICS, A NON-PROFIT CORPORATION}

The Supporting Institutions listed above contribute to the cost of publication of this Journal, but they are not owners or publishers and have no responsibility for its content or policies.

Reprinted 1966 in the United States of America 


\section{Pacific Journal of Mathematics}

\section{Vol. 11, No. 4}

A. V. Balakrishnan, Prediction theory for Markoff processes . . . . . . . . . . 1171

Dallas O. Banks, Upper bounds for the eigenvalues of some vibrating systems . . . . 1183

A. Białynicki-Birula, On the field of rational functions of algebraic groups ...... 1205

Thomas Andrew Brown, Simple paths on convex polyhedra .............. 1211

L. Carlitz, Some congruences for the Bell polynomials . . . . . . . . . . . . 1215

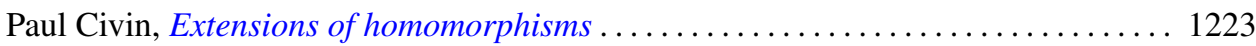

Paul Joseph Cohen and Milton Lees, Asymptotic decay of solutions of differential

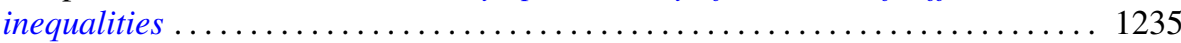

István Fáry, Self-intersection of a sphere on a complex quadric . . . . . . . . . . 1251

Walter Feit and John Griggs Thompson, Groups which have a faithful representation

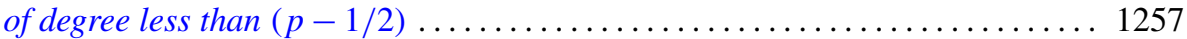

William James Firey, Mean cross-section measures of harmonic means of convex

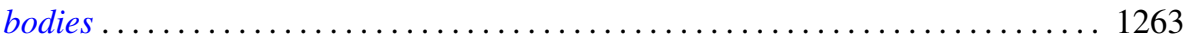

Avner Friedman, The wave equation for differential forms . . . . . . . . . . 1267

Bernard Russel Gelbaum and Jesus Gil De Lamadrid, Bases of tensor products of

Banach spaces ................................... 1281

Ronald Kay Getoor, Infinitely divisible probabilities on the hyperbolic plane . . . . 1287

Basil Gordon, Sequences in groups with distinct partial products . . . . . . . . . . . . 1309

Magnus R. Hestenes, Relative self-adjoint operators in Hilbert space . . . . . . . . . 1315

Fu Cheng Hsiang, On a theorem of Fejér ......................... 1359

John McCormick Irwin and Elbert A. Walker, On N-high subgroups of Abelian

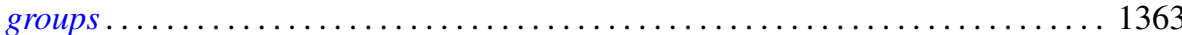

John McCormick Irwin, High subgroups of Abelian torsion groups . . . . . . . . . 1375

R. E. Johnson, Quotient rings of rings with zero singular ideal . . . . . . . . . . . 1385

David G. Kendall and John Leonard Mott, The asymptotic distribution of the time-to-escape for comets strongly bound to the solar system ...

Kurt Kreith, The spectrum of singular self-adjoint elliptic operators ....

Lionello Lombardi, The semicontinuity of the most general integral of the calculus of variations in non-parametric form ................................

Albert W. Marshall and Ingram Olkin, Game theoretic proof that Chebyshev inequalities are sharp

Wallace Smith Martindale, III, Primitive algebras with involution . . William H. Mills, Decomposition of holomorphs ..............

James Donald Monk, On the representation theory for cylindric algebras . . . . . . 1447

Shu-Teh Chen Moy, A note on generalizations of Shannon-McMillan theorem . . . . 1459

Donald Earl Myers, An imbedding space for Schwartz distributions . .

John R. Myhill, Category methods in recursion theory .........

Paul Adrian Nickel, On extremal properties for annular radial and circular slit mappings of bordered Riemann surfaces

Edward Scott O'Keefe, Primal clusters of two-element algebras . .

Nelson Onuchic, Applications of the topological method of Wazewski to certain

problems of asymptotic behavior in ordinary differential equations ...

Peter Perkins, A theorem on regular matrices................

Clinton M. Petty, Centroid surfaces .... 